conceptual difficulties exceeded the purely technical ones.

Since the book is destined to play an important part in the mathematical education of future physicists it is perhaps not inappropriate to take issue with the authors on the "philosophy" underlying their approach. Throughout history mathematics has served physics in dual capacity: (a) as a tool for obtaining numerical answers, (b) as an aid to deeper understanding and logical evaluation of the generalizations and abstractions one tends to draw from experiment and experience. Although no sharp line can be drawn between (a) and (b) the two aspects are clearly distinguishable especially if pushed to extremes. The authors have concentrated on (a) to such an extent that (b) is all but absent or lost. Thus in spite of the richness of material and the meticulous care taken in its presentation the reviewer feels that the work is inadequate as a treatise or even a textbook on mathematical methods of physics. It is a "super handbook" and an invaluable reference source, but with all its excellent features it somehow lacks cohesiveness and structure. If this book should become the "Riemann-Weber" of the twentieth century the reviewer will regretfully and nostalgically look back to the nineteenth.

MARK KAC

Primzahlen. By E. Trost. Basel, Birkhäuser, 1953. 95 pp. Paperbound, 13.50 Swiss Fr.

This little book is devoted to the elementary or nonanalytic theory of primes. Within this scope, the author has managed to cover many topics in an interesting way. There are no problems.

Among the things which are also found in almost any elementary book on the theory of numbers are the unique factorization theorem, the Fermat-Euler theorem, primitive roots, the Legendre symbol and its reciprocity law, the Möbius inversion formula, residue characters and Wilson's theorem. Also treated in some detail are the partial converse of Fermat's theorem, the representation of prime numbers by certain special forms of the kind $x^{2}+d y^{2}$, the Mersenne and Fermat numbers, prime representing functions and the calculation of $\pi(x)$ by Meissel's method.

Estimates for $\sum_{p \leqq x} 1 / p$ and the Tchebychef inequalities

$$
0<C_{1}<\pi(x) \div(x / \log x)<C_{2}
$$

are given as well as the corresponding inequalities for the functions $\vartheta(x)$ and $\psi(x)$. Also proved is Bertrand's "postulate" that there is a prime between $n$ and $2 n$; the following stronger result is also proved:

$$
0<C_{3}<\{\pi(2 n)-\pi(n)\} \div\{n / \log n\}<C_{4} \text {. }
$$


A number of sums of the type $\sum_{n \leqq x} f(n), \sum_{n \leqq x} 1 / f(n)$ for positive, increasing $f(u)$ are estimated. These, together with Abel's partial summation, enable the author to obtain the estimate

$$
\sum_{n \leqq x} \frac{\Lambda(n)}{n} \log n=\frac{1}{2} \log ^{2} x+O(\log x)
$$

and others of a similar, but more complicated, kind. These estimates prepare the way for an elementary proof of the prime number theorem. To this end, Selberg's estimate is obtained in the form

$$
\psi(x) \log x+\sum_{n \leqq x} \psi(x / n) \Lambda(n)=2 x \log x+O(x) .
$$

The subsequent proof would have been clearer if the following frequently used result had been explicitly mentioned and proved: If $a_{n} \geqq 0$ and $\sum_{n=1}^{\infty} a_{n}$ diverges, then $\sum_{n=1}^{x} o\left(a_{n}\right)=o\left(\sum_{n=1}^{x} a_{n}\right)$.

The proof of the prime number theorem which is given here is in the form due to Selberg. Inasmuch as Nagell's book gave the Erdösvan der Corput form, both proofs are now available for students. It must be stated, however, that neither of these proofs (each of which starts from Selberg's estimate) is likely to appear very transparent to beginning students. On the one hand, Selberg's argument rests on numerous partial summations (or integrations by parts), two initial estimates for $R(x)=\psi(x)-x$, and an involved deduction which leads to the self-improving inequality $|R(x)|\left\langle\alpha_{n} x\right.$ if $x>\beta_{n}$; here, $\alpha_{n+1}$ $=\alpha_{n}\left(1-c \alpha_{n}^{2}\right) \rightarrow 0$. On the other hand, Erdös' argument makes use of the limits superior and inferior, and some rather complicated splitting of summation ranges and choices of various parameters. Although one can motivate the various steps in both proofs, these are still likely to seem unnatural to the novice. Some readers will prefer the version of Selberg's proof, given in the third edition of Hardy and Wright's book, which uses integrals in place of sums.

Trost also gives the elementary proof of H. N. Shapiro that

$$
\sum_{p \leqq x, p \equiv a(m)} \frac{\log p}{p}=\frac{1}{\phi(m)} \log x+O(1)
$$

provided $(a, m)=1$. From this it follows at once that the arithmetic progression $a, a+m, a+2 m, a+3 m, \cdots$ contains an infinity of primes.

Brun's sieve and some of its applications are given. Although only the upper bound estimate is given or used, the author nevertheless seems to prefer Brun's sieve to Selberg's (which he mentions) because one can readily obtain lower bound estimates by Brun's method, 
whereas there are, to date, no published proofs for lower bounds resulting from Selberg's sieve. The author's exposition of the Brun sieve, given for the two-residue case, is one of his less successful endeavors. By means of the sieve, the convergence of the series of the reciprocals of the twin primes is proved. So also is the Schnirelmann result that each integer is the sum of a bounded number of primes.

$\mathrm{By}$ and large, the exposition is of a high order. This is particularly true of those sections in which the author gives references and résumés of known results. Such sections go beyond the usual textbook treatment of such matters and are to be commended.

On the other hand, there are typographical difficulties and more than the usual number of inaccuracies and falsehoods. The first of these arises from the author's style which frequently makes symbols appear in juxtaposition; for example, on page 40 one finds

$$
F_{n}=p 3^{(p-1) / 2} \equiv(3 / p)=-1(\bmod p)
$$

where, at the very least,

$$
F_{n}=p, \quad 3^{(p-1) / 2} \equiv(3 / p)=-1(\bmod p)
$$

would have been better. Many inaccuracies arise from the use of < in place of $\leqq$.

As for the errors, we cite the worst. Theorems 11-14 are incorrect; for example, although Theorem 11 states that an odd number of the form $4 m+1$ is a prime if and only if it is representable in exactly one way as the sum of two relatively prime squares, the simple counter-example $4 \cdot 6+1=3^{2}+4^{2}$ shows that the "if" part of this theorem is false. On page 33 , the author deduces from $x_{1}^{2}+d y_{1}^{2}=x_{2}^{2}$ $+d y_{2}^{2}$ that $x_{1}-x_{2}=d u t$ for some integers $u$ and $t$; thus he claims that $d$ divides $x_{1}-x_{2}$ whereas the example $10^{2}+12 \cdot 1^{2}=2^{2}+12 \cdot 3^{2}$, in which $d=12$ and $x_{1}-x_{2}=8$, disproves the claim. On page 56, the following statements, "proved" by the author, are incorrect:

$p_{1} p_{2} \cdots p_{\pi(n)} \sim e^{n},\{1,2,3, \cdots, n\} \sim e^{n},\left(p_{1} p_{2} \cdots p_{\pi(n)}\right)^{1 / \pi(n)} \sim n$.

Indeed, the first is equivalent to $\vartheta(n)=n+o(1)$ and hence is false; a like remark applies to the second. The correct form of the third result has the right side replaced by $n / e$ and can be deduced from $\vartheta(n)=\pi(n)\{\log n-1+o(1)\}$ or from $\pi(n)=n / \log n+n / \log ^{2} n$ $+o\left(n / \log ^{2} n\right)$ but not from $\vartheta(x) \sim \pi(x) \log x$ as the author states. On page 74 , the statement (108) is false as it stands but it can easily be made correct. And on page 90 , the result of Shapiro and Varga is incorrectly quoted as holding for all integers instead of for all sufficiently large integers. Finally, in Hilfssatz $\mathrm{C}$ on page 71 , one cannot 
conclude that $\left(y, e^{\delta / 2} y\right)$ is a subinterval of $(x, \rho x)$ since one only knows that $x \leqq y \leqq \rho x$.

Because this book is well conceived and gives a good picture of the elementary results, it is unfortunate that the execution suffers from the defects noted above. For the more mature student who reads with a skeptical eye, these defects are not of major importance but one cannot recommend this work to the unwary.

\section{LOWELL SCHOENFELD}

Entire functions. By R. P. Boas, Jr. (Pure and Applied Mathematics, vol. 5.) New York, Academic Press, 1954. $12+276$ pp. $\$ 6.00$.

This is the first book which is devoted primarily to the theory of analytic functions of exponential type, and it gives a practically complete account of the subject. A function $f(z)$ is said to be of exponential type when it is regular in an unbounded set, often the whole plane, and satisfies an inequality $|f(z)| \leqq A e^{|z|}$ on the set. Besides the theory of these functions the book contains a large chapter on entire functions in general, a chapter on the minimum modulus of an entire function, and a chapter on applications of functions of exponential type. The author and his publisher have rendered a great service by making so much material available in a single volume of moderate size and price.

One may regard Pólya, Valiron, Paley and Wiener, and S. Bernstein as the founders of the present theory of functions of exponential type. An unusually large number of mathematicians contributed to its further development (the bibliography lists close to 400 papers!). Of these, the author has probably contributed to more aspects of the theory than anybody else. Moreover, because of his past and present association with Mathematical Reviews the author was in a unique position to become thoroughly familiar with the extensive literature on the subject, including the Russian literature. In addition to these qualifications the author possesses considerable skill as an expositor, and he has thus produced a book which is both extremely useful and very readable.

The author's job has not been easy. Faced with an overwhelming amount of material and striving to be complete he had the tremendous task of ordering a veritable chaos. In the opinion of the reviewer he has succeeded exceedingly well. A good example is furnished by Theorem 8.4.16 for whose proof Levinson (1940) needed 14 pages. In the present book the theorem is just one result in an integrated theory and its proof takes only a little more than half a page. In this and other places the author's method of dealing with a 